A N N A L E S Annales de Bretagne et des Pays de l'Ouest

\title{
Nadine Vivier (dir.), Dictionnaire de la France du XIXe siècle
}

\section{Annie Duprat}

\section{(2) OpenEdition}

1 Journals

\section{Édition électronique}

URL : http://journals.openedition.org/abpo/1585

DOI : $10.4000 /$ abpo. 1585

ISBN : 978-2-7535-1487-4

ISSN : 2108-6443

Éditeur

Presses universitaires de Rennes

Édition imprimée

Date de publication : 20 septembre 2002

Pagination : 173-174

ISBN : 978-2-86847-768-2

ISSN : 0399-0826

\section{Référence électronique}

Annie Duprat, "Nadine Vivier (dir.), Dictionnaire de la France du XIXe siècle », Annales de Bretagne et des Pays de l'Ouest [En ligne], 109-3 | 2002, mis en ligne le 20 septembre 2004, consulté le 21 septembre 2020. URL : http://journals.openedition.org/abpo/1585 ; DOI : https://doi.org/10.4000/ abpo. 1585 
la vie politique tant départementale que nationale (13 fois ministre entre 1947 et 1957, année où, à la Défense nationale, il lança la construction de la spectaculaire " ligne Morice " en Algérie) une place de premier plan. Ajoutée aux éléments déjà existants sur les Jeunesses patriotes (Jean Philippet) ou les modérés, et à ceux que l'on ne manquera pas de trouver dans les thèses en cours d'achèvement de Jean-Paul Thomas et de David Bensoussan, elle laisse entrevoir la possibilité d'écrire, dans un avenir relativement proche, une histoire synthétique des droites en Loire-Atlantique depuis la Première Guerre mondiale.

Une remarque pour finir, adressée à l'éditeur : pourquoi ne pas avoir ajouté quelques pages à l'ouvrage? Elles auraient permis au lecteur de trouver des instruments de travail aussi élémentaires mais essentiels qu'un index des noms de personnes et de lieux, et une carte des cantons et des communes...

Gilles RICHARD

Nadine VIVIER (dir.), Dictionnaire de la France du XIXe siècle, Paris, Hachette, coll. " Carré Histoire ", 2002, 288 p., cartes, graphiques, bibliographie thématique et index des noms propres.

Ce dictionnaire est un instrument de travail, sobre dans sa présentation et simple dans sa conception, qui s'inscrit dans le projet éditorial d'une collection d'ouvrages destinés aux étudiants de premier cycle des universités ou des classes préparatoires. La maquette est aérée et lisible car un système de renvois permet de signaler que certains termes figurant à l'intérieur d'une notice donnent également lieu à un développement dans un autre article; quelques indispensables tableaux et schémas (l'organisation de la justice, la liste des gouvernements, les pyramides des âges, ou encore les cartes de la répartition de la population urbaine en 1806 et en 1911, par exemple) permettent de visualiser des notions abstraites et de fournir de façon très pédagogique des précisions indispensables. Le projet des auteurs, qui ont su trouver une remarquable unité d'écriture (outre Nadine Vivier qui a dirigé l'ensemble, Noëlle Dauphin, Gilles Pécout et Brigitte Waché) est résumé dans une brève introduction : mettre en évidence les spécificités d'un long siècle de l'histoire de France, dont les bornes sont, classiquement, la fin de la Révolution française, d'une part, et le début de la première guerre mondiale d'autre part.

Tous les aspects du XIX ${ }^{\mathrm{e}}$ siècle français sont présentés par un millier de notices, d'inégale longueur, en fonction d'un principe d'organisation simple : une trentaine de notices assez longues traitent d'un thème majeur et donnent lieu, par le système des renvois, à des notices brèves et précises. Prenons par exemple, dans le domaine de l'histoire religieuse, l'article " spiritualité catholique " (p. 242-243). Après un rappel de la rupture causée par la Constitution Civile du Clergé en 1790 (notice brève p. 75), les auteurs montrent l'atmosphère intellectuelle de l'époque, le matérialisme (notice brève p. 166), le romantisme (notice longue p. 225-226), exposent les aspects nouveaux ou renouvelés de la pratique du culte catholique (notices brèves, p. 229 sur la dévotion au Saint-Sacrement, p. 67 sur la pratique fréquente de la communion, p. 193 sur les pèlerinages, p. 169 sur les missions paroissiales). Mais l'étude de la transformation de la sensibilité et de la pratique religieuses ne peut faire l'économie d'un rappel des différents régimes qui se succèdent, et les renvois nous convient à faire le clair sur le Consulat (p. 75-76), la Restauration (p. 219-221) la monarchie de Juillet (p. 171- 
172) etc. À la même rubrique de la "spiritualité catholique ", sont évoquées les nombreuses " apparitions mariales " (p. 16), qui donnent elles-mêmes naissance aux " congrégations mariales" (p. 70), placées sous l'autorité des Jésuites et héritières de la reconquête catholique du $\mathrm{xVI}^{\mathrm{e}}$ siècle. Ainsi, la lecture attentive de ce dictionnaire permet de renouer le lien chronologique et intellectuel entre la France d'ancien régime et celle de l'âge industriel dans la mesure où l'on peut mieux mesurer le poids des continuités, évident dans le cas des formes de la pratique religieuse et l'importance de la dévotion mariale, illustrée par l'information mentionnée ici que le Traité de la vraie dévotion à la Sainte Vierge de Grignion de Montfort, composé en 1712, a été publié en 1842.

Les questions politiques, particulièrement importantes dans ce " siècle des révolutions ", sont largement traitées, grâce aux entrées chronologiques par type de régime évoquées plus haut, mais par la rubrique thématique portant sur les " constitutions ": de 1791 à 1875, pas moins de huit textes fondamentaux déterminent l'organisation des pouvoirs dans le pays, sur lesquels il fallait faire le point de façon précise et synthétique. On regrettera cependant que, dans le corps de la notice (p. 73-75), l'occasion ne se soit pas présentée de faire figurer le mot " charte" , ce qui aurait permis de renvoyer aux notices " charte", de 1814 et de 1830 (p. 52-53) afin de permettre à l'étudiant soucieux de repérage chronologique de bien s'y retrouver dans la succession des régimes politiques que la France a connus. Lorsque la faillite des régimes constitutionnels est patente, il arrive qu'un coup d'État les renverse : si les limites chronologiques imposées à cet ouvrage n'ont pas permis aux auteurs de prévoir une notice sur le coup d'État de brumaire (mentionné, mais sans renvoi, dans le corps de la notice sur le Consulat, p. 75), à l'inverse, le coup d'État du 2 décembre 1851 et ses conséquences immédiates donne lieu à une notice extrêmement précise (p. 78-79). La liste exhaustive qui a été établie des gouvernements de la France, de 1799 à 1914, avec l'indication des noms de quelques uns des titulaires des portefeuilles ministériels, sera d'une très grande utilité pour tous les étudiants confrontés aux explications de textes historiques (p. 125-130).

Quant à la "révolution industrielle ", notion de plus en plus difficile à définir au fur et à mesure de l'avancement des recherches, elle est traitée dans une notice (p. 224) qui renvoie elle-même à une autre brève rubrique, la " protoindustrialisation " (p. 208). Ces deux articles, qui ont le mérite de montrer de façon simple pourquoi ce concept traditionnel est largement remis en cause, renvoient à des secteurs de développement économique (la sidérurgie) mais pas aux structures du capitalisme ni aux cycles économiques, pourtant étudiés dans l'ouvrage (p. 46 pour le premier, p. 85 pour le second).

Annie DUPRAT

Martine Sonnet, Brigitte Keriven, Claude Ghiati, Isabelle Havelange, Bibliographie annuelle de l'histoire de France du ve siècle à 1958. Année 2000, t. 46, CNRS éditions, Paris, 2001, 1054 p., $102 €$.

On ne présente plus cet indispensable outil de travail dont la parution annuelle, depuis près d'un demi-siècle, est régulièrement saluée par les historiens. Les quatre ingénieures du CNRS ("Institut d'histoire moderne et contemporaine ") qui ont réalisé cette quarante-sixième livraison de l'ouvrage à partir " principalement " des collections de la Bibliothèque Nationale de France ont 